\title{
The dynamics of ochratoxigenic fungi contents through different stages of dried grape production
}

\author{
Lusine Hakobyan ${ }^{1}$, Karine Grigoryan ${ }^{2}$, and Armen Trchounian ${ }^{1,2}$ \\ ${ }^{1}$ Department of Biochemistry, Microbiology and Biotechnology, Yerevan State University; 1 Alex Manoogian Str., Yerevan 0025, \\ Republic of Armenia \\ ${ }^{2}$ Research Institute of Biology, Yerevan State University, 1 Alex Manoogian Str., Yerevan 0025, Republic of Armenia
}

\begin{abstract}
Dried vine fruit (raisin, sultana and currant) is the second (after wine) most important product of viticulture. Concerning this, the contamination of dried grape by ochratoxigenic fungi and ochratoxin A (OTA) has attracted much attention. Favorable climatic conditions in countries with well-developed viticulture contribute to the spreading of ochratoxigenic fungi. The aim of this work was to identify the contamination sources of dried vine fruit by ochratoxigenic filamentous fungi and OTA, as well to determine the Critical Control Points $(\mathrm{CCP})$ at different stages of production. Primary contamination of grapes occurred during vegetation, especially maturation period, when the risk of mechanical damages was the highest one. 48 samples of soil and 81 samples of fresh grape berries collected in 4 regions of Armenia were investigated. As a result, 22 micromycetes sp. from 7 genera were isolated. Drying process is one of the main CCP. As the most of dried products is produced by open sun drying method, secondary contamination occurs in plants. In our studies 27 species of filamentous fungi were revealed in 87 samples of dried vine fruit, collected at different stages of production. The samples had quite high contamination level by potential toxigenic $A$. niger and $A$. carbonarius species.
\end{abstract}

\section{Introduction}

The quality of dried grapes mainly depends on the parameters of microbiological safety, which include pathogenic and toxigenic microorganisms contaminating dried vine fruit. Dried vine fruits are often contaminated by filamentous fungi, the presence of which in food is a danger to the health of the consumer. Therefore, the content of filamentous fungi and some other microorganisms in food is normalized in SanPiN2.3.2.1078-01 [1].

Dried fruits, including dried vine fruits, are susceptible to contamination by fungi and mycotoxins, since they contain a sufficient amount of moisture and sugars [2]. Aspergillus, Penicillium, Fusarium and Alternaria are the main genera of filamentous fungi contaminating food including dried vine fruits [3].

The problem of contamination of dried vine fruit by ochratoxin A has been studied in many countries with developed viticulture and winemaking such as Spain [4], France [5], Italy [6], and Australia [7]. In these studies, it is claimed that most of the ochratoxigenic strains belong to the species A. carbonarius. A few numbers of strains belonging to $A$. niger species which synthesized OTA. In Hungary among species isolated from raisin samples in addition to A. niger and A. caorbnarius another ochratoxigenic species of $A$. tubingens dominated [8].

In countries of South America the main contaminants of grapes, raisins and other grape derived products are species of A. niger, A. ochraceus, A. carbonarius and Alternaria alternate [9]. In Argentina, the most common species was A. niger, but only a few number of isolated strains of this species produced OTA. A. carbonarius was revealed more rarely but $82.6 \%$ of the strains in one case [10] synthesized OTA. Toxigenic species A. ochraceus and A. japonicus, which synthesized OTA, were also detected.

Studies show that in California the presence of ochratoxin A in raisin samples is also associated mainly with the species A. carbonarius [11].

Analyzes conducted in countries of the Middle East region (Saudi Arabia, Yemen and Iraq) have shown the similar results. The species of A. niger dominated in all studied samples from three countries. This species was the main ochratoxigenic species in samples from Iraq [12]. A. carbonarius also revealed frequently (60.3\%) [13] and the frequency of occurrence of A.niger was up to 92.3\%. Among the species isolated from raisin samples in Saudi Arabia, the fungi from genera Aspergillus, Alternaria, Cladosporium, Fusarium and Rhizopus, were also extended [14]. In addition to A.niger the toxigenic species of A. flavus, A. fumigatus andA. ochraceus from genus Aspergillus [15] were often isolated from raisin samples in Yemen Republic.

In order to reduce the risks of contamination of the final product in dried vine fruit production much attention is paid to the development of technologies and methods of inhibition the growth and development of micromycetespotential producers of mycotoxins. The practice of drying varies depending on the geographical location and varieties of grapes [16]. There are three main methods that are used in production of dried vine fruits: open sun drying (or open air drying), shadow drying and mechanical drying. Traditional open sun drying method has been used for thousands of years in Asia and other countries around the world. More than $90-95 \%$ of dried products 
produced in all countries of the world are prepared by the open sun drying method. Along with the traditional sun drying method, another drying method on vine (DOV) is used. This method is especially common in Uzbekistan and USA. In order to prevent the development of mold fungi and synthesis of mycotoxins, it is advisable to use solar dryers that accelerate the process and simplify the control of drying conditions [17]. Recently, in America various methods of mechanical drying of grapes have been developed, in which the risk of effluence of unfavorable weather conditions as well contact of workers with raw materials and final products, are reduced [18]. The basis of these methods is the mechanization of all processes: harvesting, drying and collecting of final dried products.

The use of appropriate agrotechnical measures and fungicides in grapes cultivation can significantly prevent the growth of fungi from genus Aspergillus and contamination of final product with ochratoxin A [19]. After harvesting, the most effective method for limiting the development of mold fungi is the treatment of raw grape with sulfur dioxide $\left(\mathrm{SO}_{2}\right)$ [20]. $\mathrm{SO}_{2}$ and its derivatives have long been used for treatment of raw grape, as a preservative inhibiting the development of mold-forming fungi and other microorganisms. It also acts as an antioxidant, which prevents oxidation processes and lead to the changes of the color of final product.

\section{Materials and methods}

\subsection{Sampling}

87 dried vine fruit samples (white and black varieties of raisin and sultana) were analyzed. 39 samples were collected from different markets and supermarkets of Yerevan, and 48in some manufactures in Armavir region in accordance with GOST 1750-86 [21] and Commission Regulation (EC) No 401 [22]. Samples were collected at different stages of production: raw materials, the pretreated material - after blanching (alkali treatment), after treatment by $\mathrm{SO}_{2}$, during drying process (2-4 weeks), after drying and during storage.

48 soil samples were taken in vineyards in different depths under vine $(5 \mathrm{~cm}, 15 \mathrm{~cm}$ and $25 \mathrm{~cm}) .81$ samples of fresh white and black grape were analyzed. The sampling of soils, vegetative organs of vine and grape fresh grape berries was done during harvesting in four regions of Armenia: Ararat, Armavir, Kotayk and Vayots-Dzor. In total 225 samples were studied.

\subsection{Mycological analysis of dried vine fruit, grape berries and soil samples}

Mycological analyses of dried vine fruit samples were carried out with direct planting and serial dilution planting methods [23].The dilution includes the following steps: 1) shaking the product suspension for 15 minutes on a shaker; 2) infusion for 10 minutes; 3) preparation of serial dilutions $1: 10$ and $1: 100 ; 4)$ surface plating from dilutions on nutrient mediums. Plates were incubated at $25^{\circ} \mathrm{C}$ for 7 days. Following nutrient mediums were used: CYA (Chapek-Yeast Agar medium, HiMedia Ltd.), GYA (Glucose-Yeast Agar medium, HiMedia Ltd.), and MEA (Malt-Extract Agar medium, HiMedia Ltd.).

In order to inhibit the growth of superficial saprotrophic mycobiota and reveal the internal mycoflora of dried vine fruit, fresh grape berries and vegetative organs of vine, the samples were exposed to external sterilization before analysis. For this the samples were treated with $3 \%$ solution of sodium hypochlorite for 15 minutes, after which the samples were washed with distillated water.

The aim of this work was to identify the contamination sources of dried vine fruit by ochratoxigenic filamentous fungi and OTA, as well to determine the Critical Control Points (CCP) at different stages of their production.

\subsection{Identification of fungi species}

Identification of isolated cultures of filamentous fungi was done based on macroscopic and microscopic characteristics by following manuals: [24-27]. For identification of species from section Aspergillus Nigri cultures were grown on Czapek Yeast Extract Agar (CYA) at $25 \pm 1^{\circ} \mathrm{C}$ and $37 \pm 1^{\circ} \mathrm{C}$. Fungi species are forming characteristic colonies and retain the ability to generate exudate and pigmentation on this nutrient medium.

\subsection{Data analysis}

The quantity of microscopic fungi in $1 \mathrm{~g}$ food was detected according to NF ISO 7698-91 [28] using the following formula:

$$
\omega=\frac{\sum \mathrm{C}}{\left(\mathrm{n}_{1}+0.1 \mathrm{n}_{2}\right) \mathrm{d}}
$$

in which

$\sum \mathrm{C}$-sum of colonies of fungi grown in all dishes; $\mathrm{n}_{1}$ - number of dishes used for I dilution; $\mathrm{n}_{2}-$ number of dishes used for II dilution; $\mathrm{d}-$ dilution coefficient.

The frequency of occurrence of fungi species was determined by the ratio of quantity of samples, in which the species was revealed, to the total quantity of analyzed samples [29]:

$$
\mathrm{A}=\mathrm{B} / \mathrm{C} * 100 \% \text {, }
$$

in which

$\mathrm{B}$ - number of samples in which species was detected; $\mathrm{C}-$ total number of analyzed samples.

\section{Results and discussion}

\subsection{Filamentous fungi from section Aspergillus Nigri contaminating dried vine fruit}

Mycological analysis of 87 dried vine fruit samples were carried out. 48 samples were collected immediately in plants and 39 in different markets and supermarkets in Yerevan.

The results showed that the contamination level of dried vine fruits by filamentous fungi $\left(2.2 \times 10^{2}-\right.$ $4.3 \times 10^{4} \mathrm{CFU} / \mathrm{g}$ ) often exceeds the maximum permissible level of this parameter presented in local and international sanitary rules and regulations.

According to SanPin 2.3.2.1078-01 maximum permissible amount of diaspora of molds in $1 \mathrm{~g}$ of dried vine fruit is $5 \times 10^{2} \mathrm{CFU} / \mathrm{g}$.

27 species of filamentous fungi belonging to 5 genera: Mucor, Aspergillus, Penicillium, Trichoderma andAlternaria, were isolated and identified. 13 (48\%) 


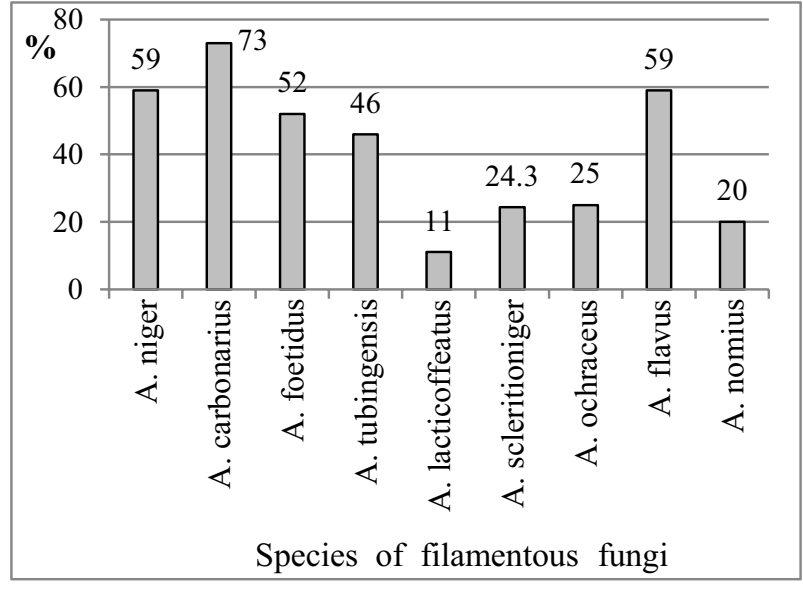

Figure 1. The frequency of occurrences of toxigenic species from genus Aspergillus in dried vine fruit.

species of the total amount of isolated filamentous fungi belong to the genus Aspergillus.

Contamination of dried vine fruits by potentially toxigenic species $A$. niger and A. carbonarius is quite high. This species are dominant and hav 59\% and $73 \%$ of frequency of occurrence respectively. Monopolistic growth of A. niger was often observed during mycological analysis of samples. Some strains of this species are characterisied by high antagonistic activity and high energy of growth.

The results of scientific research conducted in a number of countries have shown that the detection of ochratoxin A in vine derived products, including dried vine fruits, is associated first of all with the presence of $A$. carbonarius species [10,11].

Species A. sclerotiicarbonarius (34.7\%), A. tubingensis (46\%), A. foetidus (52\%) and A. flavus (59\%) had an average frequency of occurrence (Fig. 1). These species except A. flavus belong to sectionAspergillus Nigri.

A. sclerotioniger and A. lacticoffeatus species, known as potential producers of OTA, were revealed on the new substrate - dried vine fruit for the first time in our investigations. According to Samson [27] species A. sclerotioniger, A. lacticoffeatus and A. niger are also potential producers of ochratoxin A. However, only certain strains of these species are capable to produce mycotoxins.

Nine potentially toxigenic fungi species of genus Aspergillus were revealed in samples of dried vine fruit. Seven of these species are ochratoxigenic, 6 of which belong to the section A. Nigri (A. niger, A. carbonarius, A. tubingensis, A. foetidus, A. sclerotioniger, A. lacticoffeatus)and one to the section A. Circumdati (A. ochraceus). A. flavus and A. nomius species belong to the sectionAspergillus Flavi. These fungi are potential producers of aflatoxins.

Investigations of numerous authors [4,6-8] proved that A. niger, A. carbonarius, A. flavusand A. tubingensis species from genus Aspergillus are the specific microflora of dried vine fruit.

\subsection{Mycobiota of soil samples from vineyards in different regions in Armenia}

The main sources of contamination of dried vine fruit, wine and other grape processing products by species A. carbonarius and A. niger, is the soil and its residues

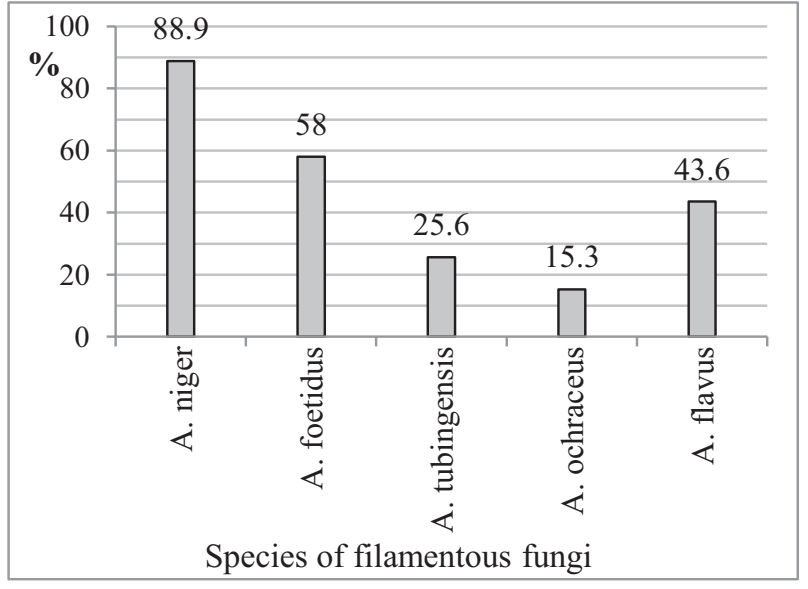

Figure 2. The frequency of occurrences oftoxigenic species isolated from soil samples.

on grape berries. In order to identify the sources of contamination of Armenian dried vine fruit by toxigenic species, mycological analysis of 48 samples of soil from vineyards in four regions in Armeniawas carried out.

As a result of the study, 22 species of filamentous fungi from 7 genera: Aspergillus, Penicillium, Alternaria, Fuzarium, Cladosporium, Trichoderma andMucor, were isolated and identified. 10 species were revealed in analyzed samples of soil belonged to the genus Aspergillus and 6 - to the genus Penicillium. Species of ochratoxigenic fungi A. niger (88.9\%), A. foetidus (58\%) and A. tubingensis (25.6\%), had high frequency of occurrence (Fig. 2).

A. carbonarius species was not revealed in any of analyzed soil samples. Other species producers of ochratoxin A from section Aspergillus Nigri were isolated from the surface layers of soil more often than from deeper layers. High contamination level of surface layers soil by micromycetes - producers of ochratoxin A is revealed during ripening and harvesting. In this period grape berries contain high quantity of glucose and moisture. Implementation of Good Agricultural Practises in vineyards is important to reduce the risks of main contamination of grape berries as the row materials.

\subsection{Mycological analysis of fresh grape berries}

Contamination of grape by filamentous fungi often occure due to the presence of mechanical damages on the skin of grape berries throuth which micelium of fungi penetrate inside. More favorable conditions for the growth of fungi are created in ripened berries, in which $\mathrm{pH}$ value is low and a sugar content is high.

Grape berries in the vegetation season and especially harvesting period are mainly affected by saprotrophic fungi from genera: Penicillium, Aspergillus, Fuzarium, Cladosporium and Alternaria.

We analyzed 81 samples of white and black varieties of grape cultivated in 4 regions in Republic of Armenia during the harvest season. As a result of the mycological analysis of fresh grape berries 19 species of micromycetes from 5 genera: Aspergillus, Penicillium, Alternaria, Cladosporium and Mucor, were isolated and identified [30]. 


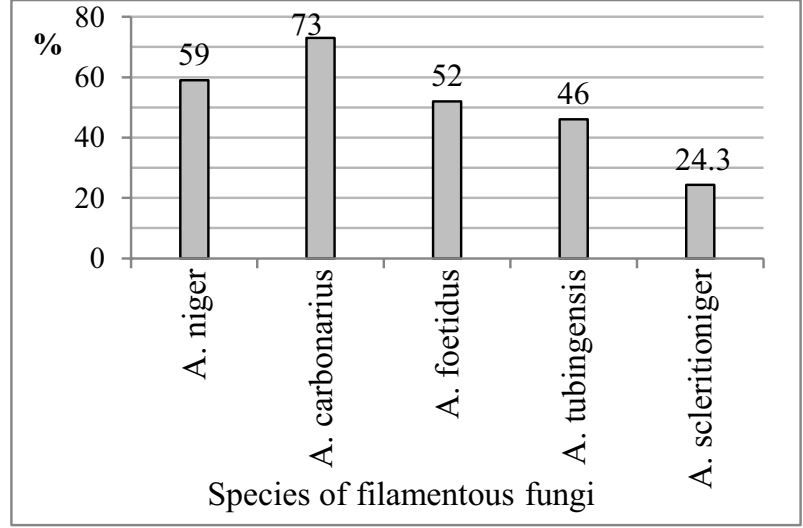

Figure 3. The frequency of occurrence of fungi species from section Aspergillus Nigri contaminating Armenian grape.

Table 1. Contamination levels of grape samples collected in four regions in Armenia.

\begin{tabular}{|l|c|c|}
\hline Region & $\begin{array}{c}\text { Contamination } \\
\text { level in CFU/g }\end{array}$ & $\begin{array}{c}\text { Number of } \\
\text { isolated species }\end{array}$ \\
\hline Armavir & $65-2 \times 10^{2}$ & 13 \\
\hline Ararat & $60-95$ & 18 \\
\hline Kotayk & $80-2.8 \times 10^{2}$ & 16 \\
\hline Vayotsdzor & $40-90$ & 9 \\
\hline
\end{tabular}

Table 2. Fungi species isolated from fresh grape berries.

\begin{tabular}{|l|c|c|c|c|}
\hline \multirow{2}{*}{ Species } & \multicolumn{4}{|c|}{ Frequency of occurrence of fungi (\%) } \\
\cline { 2 - 5 } & $\begin{array}{c}\text { Armavir } \\
\text { region }\end{array}$ & $\begin{array}{c}\text { Ararat } \\
\text { region }\end{array}$ & $\begin{array}{c}\text { Kotayk } \\
\text { region }\end{array}$ & $\begin{array}{c}\text { Vayotsdzor } \\
\text { region }\end{array}$ \\
\hline Aspergillus niger & 41 & 36 & 49.5 & 29 \\
\hline A. carbonarius & 45.7 & 24 & 48.3 & 8 \\
\hline A. foetidus & 36 & 21 & 5.4 & 11 \\
\hline A. sclerotioniger & 14 & 10.4 & 2 & - \\
\hline A. tubingensis & 7.8 & 6.3 & 4.3 & 2.3 \\
\hline A. japonicus & - & 4 & 4 & 5 \\
\hline A. uvarum & 3.9 & 4.2 & 2.4 & - \\
\hline A. aculeatus & 2 & 3.4 & 1.5 & - \\
\hline A. pulverulentum & - & 2.6 & 1.5 & 3 \\
\hline A. flavus & 9 & 11 & 17 & - \\
\hline
\end{tabular}

8 species belonged to the section Aspergillus Nigri. The frequencies of occurrence of these species are presented in Fig. 3. From potentially ochratoxigenic fungiA. niger, A. carbonarius, A. foetidus, A. sclerotioniger and $A$. tubingensis species, were identified.

Species A. carbonarius, A. niger, and A. foetidus dominated in all analyzed samples. A. flavus species had an average frequency of occurrence.

Comparative mycological analysis of grape berries, selected in 4 regions, shown that grape samples from VayotsDzor region had the lowest degree of contamination: 40-90 CFU/g (Table 1).

Only 6 species from genus Aspergillus were revealed and identified in these samples (Table 2). And the content of potentially toxigenic fungi was quite low in samples from VayotsDzor region.

The results of mycological analysis grape and soil samples collected in the vineyards allowed us to identify the main sources of contamination of dried vine fruit by A. carbonarius and A. niger ochratoxigenic species. Thus, grape berries affected by the "black" mold are the main

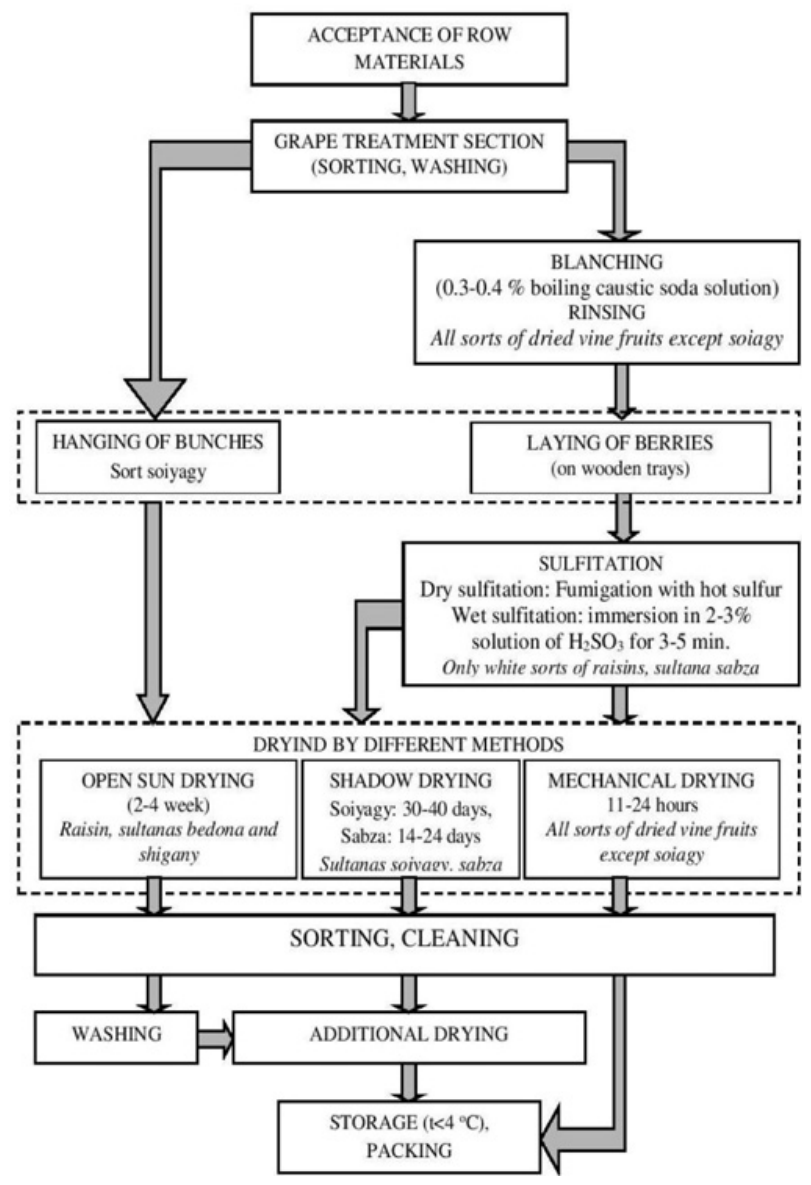

Figure 4. Main technological stages of different dried vine fruit production.

source of contamination of dried grapes by ochratoxigenic species from genus Aspergillus.

\subsection{The influencce of drying technologies on growth and evolution of filamentous fungi in dried vine fruit}

Technological regimes of grape drying also significantly affect the contamination level of the final product by fungi and mycotoxins. During open air drying the risk of contamination of dried product by mold fungi and mycotoxins increases. These occurres due to high probabilities of mechanical damage on grape berrie skin. The main schem of dried vine fruit production is given in Fig. 4.

During drying period the contamination degree of dried vine fruits directly depends on the use of sulphitation and the content of $\mathrm{SO}_{2}$ in the intermediate and final products. The latter is an effective preservative and has a significant inhibitory effect on the development of mycelial fungi, including toxin-producing species, during drying and storage.

Some authors investigated the influence of drying technologies on contamination of the processing and final product by filamentous fungi and mycotoxins [31].

In Armenia, the main part of dried foods produced by traditional open sun drying method. But along with this solar dryers are also used. CCPs are reduced during production of dried vine fruit in this dryers. 
Table 3. Contamination level (CFU/G) of dried vine fruit at different stages of production.

\begin{tabular}{|l|c|c|c|c|c|c|}
\hline $\begin{array}{l}\text { Number of } \\
\text { analyzed } \\
\text { samples }\end{array}$ & Samples & Raw material & $\begin{array}{c}\text { After } \\
\text { treatment } \\
\text { bulfur dioxide }\end{array}$ & $\begin{array}{c}\text { During } \\
\text { drying }\end{array}$ & After drying & $\begin{array}{c}\text { During storage } \\
\left(\mathbf{a t}+\mathbf{8}^{\circ} \mathbf{C}\right.\end{array}$ \\
\hline 12 & White raisin & $80-1.4 \times 10^{2}$ & 0 & $2.4 \times 10^{2}-3 \times 10^{2}$ & $4.5 \times 10^{2}-6.8 \times 10^{2}$ & $4 \times 10^{2}-7 \times 10^{2}$ \\
\hline 12 & White sultana & $90-1.3 \times 10^{2}$ & 0 & $95-2 \times 10^{2}$ & $2.2 \times 10^{2}-5.2 \times 10^{2}$ & $2 \times 10^{2}-5.5 \times 10^{2}$ \\
\hline 12 & Black raisin & $70-1.8 \times 10^{2}$ & $*$ & $5.2 \times 10^{2}-2 \times 10^{3}$ & $2.6 \times 10^{3}-1.8 \times 10^{4}$ & $2.3 \times 10^{3}-2 \times 10^{4}$ \\
\hline 12 & Black sultana & $1.5 \times 10^{2}-2 \times 10^{2}$ & $*$ & $1.8 \times 10^{2}-8.5 \times 10^{2}$ & $2.6 \times 10^{3}-3.4 \times 10^{3}$ & $2.6 \times 10^{3}-4 \times 10^{3}$ \\
\hline
\end{tabular}

* Sulfur dioxide treatment is not permissible in manufacture of these dried vine fruit varieties.

We carried out mycological analysis of 48 samples of Armenian dried vine fruit at all stages of manufacturing in order to determine the sources of contamination of the final product by filamentous fungi and identify stages in which the product is most prone to risk of contamination.

Samples were collected in two manufacturs in Armavir region at different stages of production: raw materials, the pretreated material - after blanching (alkali treatment), after treatment by $\mathrm{SO}_{2}$, during drying process (2-4 weeks), after drying and during storage.

Studies have shown that the contamination level of processing product increased during the entire production process (Table 3). However, no fungi growth was detected in the samples taken immediately after treatment by $\mathrm{SO}_{2}$, while the high contamination degree of the final product was observed. This proves an inhibitory effect of $\mathrm{SO}_{2}$ on the growth and evolution of filamentous fungi. Immediately after sulfitation process $\mathrm{SO}_{2}$ content in the intermediate product is in the maximum level.

As the most quantity of dried vine fruit is produced by the open sun drying method, so a secondary contamination occurs. Analyses of samples taken in this stage of production showed a high contamination level of intermediate product by filamentous fungi. In some cases the value of $\mathrm{CFU} / \mathrm{g}$ exceeded the maximum permissible $\left(5 \times 10^{2}\right)$. Only contamination levels of the samples dried in closed solar dryers were within the normal range.

As the black varieties of dried vine fruit had not been treated with sulfur dioxide, so their contamination levels were higher than white varieties. Concerning this the blanching stage of these products, which is not followed by sulfitation, becomes a Critical Control Point. The reason of this are microcracks on the skin of grape berries formed due to the blanching.

Contamination of product by toxigenic species increases during grape drying process. The relative proportion of $A$. carbonarius in processing grape increases as the humidity reduces in grape berries (Fig. 5).

In comparison with open air drying method the mechanical drying in closed chambers, with controlled temperature and humidity, provides the mycological safe final product. Grape drying process is often carried out at a temperature of $50^{\circ} \mathrm{C}$. Under such a temperature regime, the probability of OTA biosynthesis is minimal, since the growth of $A$. carbonarius is almost completely inhibited [32]. However, a sharp decrease of the content of vitamins, polyphenols, anthocyanins, ensuring the quality and nutritional value of final product, is observed at this temperature.

Vacuum drying method is currently used in dried vine fruits production for obtaining a safe product for the consumer.Independence with the used drying technology,

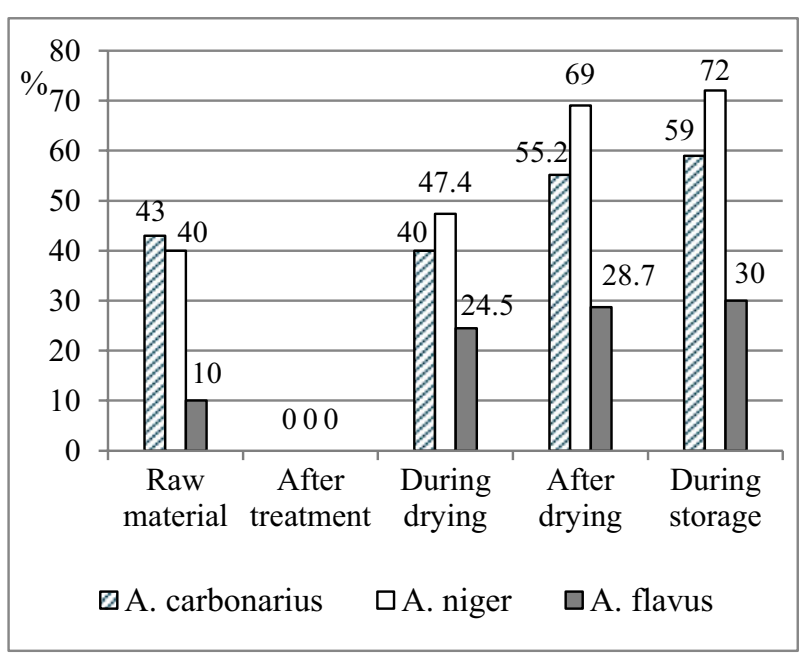

Figure 5. Percentage content of toxigenic species of fungi in different stages of production.

hygienic conditions is essential in all stages of dried vine fruit manufacturing: starting from grape harvest to the drying process, transport, packaging and storage. The absence of these conditions can lead to the secondary contamination and cross contamination of raisins by OTA-producing fungi.

\section{Conclusions}

Contamination of dried vine fruit by species from Aspergillus niger section mainly occurs during the cultivation of grape: in ripening and harvesting stages. Secondary contamination by fungi species can occur in case of bad sorting and drying process, and violation of drying and hygienic conditions in manufactures.

Climatic conditions playe an important role during production by open sun drying method. In rainy weather, the probability of secondary contamination of the intermediate product by toxigenic fungi increases.

The Critical Control Points (CCP) in production of dried vine fruits are raw grape storage, transportation conditions to the manufactur plant and technology of pretreatment of raw materials before drying.

For prediction of contamination degree of dried products by mycotoxins some aspects must be taken into account, such as weather conditions before harvest, types and amounts of fungicides used during vegetation, moisture content of grape berries during harvest and contaminant species of fungi. It is necessary to use unaffected, benign raw materials for reducing the risks of contamination of raw materials by mycotoxigenic species from genus Aspergillus and the accumulation 
of mycotoxins in dried vine fruits. Special attention should be given to the prevention of the contamination of vineyards by ochratoxigenic fungi. This can be done by carrying out hygienic measures in the vineyard areas. It is also necessary to follow the basic principles of Good Agricultural, Industrial and Hygienic Practices (GAP, GMP, GHP).

The drying process is the next important step to prevent the contamination of the final product. Our studies have shown that at this stage of production, the risk of contamination of the product by fungi increases sharply. To ensure the microbiological safety of the dried grape produced, it is important to determine CCP in plants or in storage houses. This will help manufacturers to develop and apply a suitable HACCP system that will eliminate unforeseen sources of contamination of products by mycotoxins. At this stage of production, the Critical Control Points are: pests and insects, drying time, frequency of turning grape berries, hygienic conditions, and weather conditions (temperature, humidity, rain, etc.). The latter is especially important in the production by open air drying method. Control measures should be applied at all critical points. For each identified CCP, the measured parameters must be defined and their critical limits must be established.

\section{References}

[1] SanPiN 2.3.2.1078-01, Sanitary-epidemiological rules and regulations. Production row materials and foodstuffs. Hygienic requirements for the safety and nutritional value of food, 180 page (2001)

[2] S.M.S. Alghalibi, A.R.M, Assiut University Bulletin of Environmental Research 7(2), p. 26-30 (2004)

[3] J.I. Pitt, A.D. Hocking, Fungi and Food Spoilage, 3rd ed., Springer, p. 413-414 (2009)

[4] M. Bau, M.R. Bragulat, M.L. Abarca, S. Minguez, F.J. Cabanes, Int J. Food Microbiol. 98, p. 125-130 (2005)

[5] L. Sage, S. Krivobok, E. Delbos, F. Seigle-Murandi, E.E. Creppy, J. Agric. Food Chem 50, p.1306-1311 (2002)

[6] P. Battilani, P. Giorni, T. Bertuzzi, S. Formenti, A. Pietri, Int. J. Food Microbiol. 111, p. S53-S60 (2006)

[7] S.L. Leong, A.D. Hocking, J.I. Pitt, B.A. Kazi, R.W. Emmett, E.S. Scott, Int. J Food Microbiol. 111(1), p. S10-S17 (2006)

[8] J. Varga, S. Kocsube, Z. Koncz, J. Teren, Actaalimentaria. 35(3), p. 289-294 (2006)

[9] S.N. Chilze, C.E. Magnoli, A.M. Dalcero, Int. J. Food Microbiol. 111(1), S5-9 (2006)

[10] S.M. Romero, R.M. Comerio, G. Larumbe, A. Ritieni, Int. J. Food Microbil. 104(1) 43-49, (2005)
[11] J.D. Palumbo, T.L. O'Keeffe, S.J. Vasquez, N.E. Mahoney, Let. in Appl. Microbiol. 52, p. 330-336 (2011)

[12] M.T. Farid, A.L. Neveen Nowzad Rajab, Zan. J. of Pure and Appl. Sci. 26(4), pp. 49-60 (2014)

[13] A.A.M. Saadullah, S.K. Abdullah, 7(1), pp. 61-68 (2015)

[14] R.M. Gashgari, Y.M. Shebany, Y.A. Gherbawy, Fborn. Pathog. and Dis. 8(11), p. 1221-1227 (2011)

[15] S.M.S. Alghalibi, A.R.M. Shater, Assiut Uni. Bulle. Of Envir. Res. 7(2), p. 26-30 (2004)

[16] C. Domingo, Li. Giralt, Gener. Catalun. Inst. Cat. Vin. 1, 27 (2006)

[17] D.R. Pangavhane, R.L. Sawhney, P.N. Sarsavadia, Ener. Convers. Manag. 43(1), p. 45-61 (2002)

[18] R.L., Williams, S.A. Shepard, J.E. Casey, Calif. Agri. Tech. Inst., 74 p (2002)

[19] L. Covarelli, G. Beccari, A. Marini, L. Tosi, Food Cont. 26, p. 347-356 (2012)

[20] A.D. Hocking, Xerophilic fungi in intermediate and low moisture foods, (New York: Marcel Dekker Inc. 3, 1992)

[21] Gosstandart of the USSR, Dried fruits. Acceptance rules, methods of analysis (1986)

[22] Commission Regulation (EC) No 401/2006, Offic. J. EU, L70, p. 12-34 (2006)

[23] JI.Pitt, A.D. Hocking, Fungi and Food Spoilage, (2nd Ed. Black. Acad. and Prof., London 1997)

[24] V.I. Bilay, E.Z. Koval, Aspergilles, (Kiev, Naukova Dumka, 1988) (in Russian)

[25] K.B. Raper, D.I. Fennell, The genus Aspergillus. (Robert E. Krieger Publ. Co. Inc. Florida, USA, 1977)

[26] R.A. Samson, J.A. Houbraken, F.A. Kuijpers, J.M. Frank, J.C. Frisvad, In Stud. Myc. 50, 45-61 (2004)

[27] R.A. Samson, P. Noonim, M. Meijer, J. Houbraken, J.C. Frisvad, J. Varga, In Myc. 59(13), 129-145 (2007)

[28] NF ISO 7698-91.Controle de la qualite des produits alimentaires controle microbiologique. Direktives generales pour le denombrement des levures et moisissures (1993)

[29] I.A. El-Kady, J.N. Abdel-Hafez, S.S. El-Maraghy, In J. Mycopath. 77, p. 103-109 (1982)

[30] K. Grigoryan, L. Hakobyan, M. Sargsyan, J. Hyg. Engin. Design, p. 97-100 (Ohrid, Republic of Macedonia, 2011)

[31] A.W.S. Kassem, M.A. Al-Sulaiman, A.E.M. Aboukarima, S.S. Kassem, Austr. J. Bas.\& Appl. Scien. 5(6), p. 230-241 (2011)

[32] D. Spadaro, S. Patharajan, A. Lore, M.L. Gullino, Garibaldi A. Phytopathol. Mediterr. 49, p. 65-73 (2010) 